\title{
Numerical Simulation of Fluid Flow and Heat Transfer in a DC Non-Transferred Arc Plasma Torch Operating Under Laminar and Turbulent Conditions*
}

\author{
DENG Jing (邓晶), LI Yaojian (李要建), XU Yongxiang (徐永香), \\ SHENG Hongzhi (盛宏至) \\ Institute of Mechanics, Chinese Academy of Sciences, Beijing 100190, China
}

\begin{abstract}
In this work, a magnetic fluid dynamics (MHD) model is used to simulate the electromagnetic field, heat transfer and fluid flow in a DC non-transferred arc plasma torch under laminar and turbulent conditions. The electric current density, temperature and velocity distributions in the torch are obtained through the coupled iterative calculation about the electromagnetic equations described in a magnetic vector potential format and the modified fluid dynamics equations. The fluid-solid coupled calculation method is applied to guarantee the continuity of the electric current and heat transfer at the interface between the electrodes and fluid. The predicted location of the anodic arc root attachment and the arc voltage of the torch are consistent with corresponding experimental results. Through a specific analysis of the influence of mass flow rates and electric current on the torch outlet parameters, the total thermal efficiency, thermal loss of each part, and the laws of the variation of outlet parameters with the variation of mass flow rates and electric current was obtained. It is found that operation under a laminar condition with a limited area of the anode could increase the total thermal efficiency of the torch.
\end{abstract}

Keywords: modeling, arc plasma torch, MHD, arc root, fluid-solid coupled method PACS: $52.65 .-y$

\section{Introduction}

DC arc plasma torches are used to generate thermal plasma jets in different industrial applications, such as thermal plasma waste treatment ${ }^{[1]}$, atmospheric or low-pressure plasma spraying ${ }^{[2]}$, plasma-assisted chemical vapor disposition, plasma preparation of ultra-fine powder ${ }^{[3]}$, etc.

It is important to understand the arc physics inside the plasma torch to improve the application of thermal plasma technique. Because the properties of heat transfer and fluid flow inside the torches is hard to observe and measure, the numerical simulation method is widely applied. Through the coupled iterative calculation about electromagnetic equations and modified fluid dynamics equations, the electric potential, electric current density, magnetic field intensity, temperature and velocity distributions in the torch can be obtained. In recent years, electromagnetic equations in magnetic vector potential format are usually adopted in the numerical study of the plasma arc ${ }^{[4]}$ and torches ${ }^{[3,5,6]}$. Compared with the traditional method that solves magnetic field intensity by calculating the integral of electric current density according to Ampere circuit theorem ${ }^{[7]}$, the new method with magnetic vector potential is much more effective, which can simplify the efforts in modifying the numerical solver and save the CPU time dramatically.

The jets produced by the DC arc plasma torches show a variety of features with different electric cur- rent, mass flow rate of working gas, structural design of torches, species of surrounding gas (single component or multi-component) and its pressure. The plasma jets can be basically classified as laminar jets and turbulent ones by the state of flow. The corresponding measured results indicated that a laminar jet will transit to a turbulent jet when the mass flow rate increases with the same electric current. In addition, the increase of current can restrain the transitional process ${ }^{[2]}$.

The related experimental results exhibited the phenomena that the property of the jets will change with the mass flow rates and the electric current of the torch. However, the specific influencing factor and the laws of the variation are not clear yet. In this work, the variation laws will be studied by simulating and analyzing the inner fields and the outlet parameters of a plasma torch under different operational conditions. Moreover, increase of the thermal efficiency of the plasma torch is very important to save electric energy, so both thermal efficiency and thermal loss of each part of the torch will be studied for searching better operational conditions and optimizing the structural design.

\section{Numerical simulation methods}

\subsection{Assumptions}

The assumptions made in the present modeling include:

\footnotetext{
*supported by National Natural Science Foundation of China (No. 50476081)
} 
a. The plasma flow is steady and axially symmetric;

b. The arc column region is assumed to be in a local thermodynamic equilibrium (LTE) state;

c. The plasma is optically thin;

d. The density, specific heat, viscosity and both thermal and electrical conductivities of the arc plasma are functions of temperature only;

e. The gravity, viscous dissipation, and pressure work are neglected.

\subsection{Governing equations and modeling method}

In the steady and axial-symmetric conditions, the MHD equations describing arc plasma motion are as follows:

Mass conservation:

$$
\frac{1}{r} \frac{\partial}{\partial r}\left(r \rho v_{r}\right)+\frac{\partial}{\partial z}\left(\rho v_{z}\right)=0
$$

Radial momentum conservation:

$$
\begin{gathered}
\frac{1}{r} \frac{\partial}{\partial r}\left(r \rho v_{r}^{2}\right)+\frac{\partial}{\partial z}\left(\rho v_{r} v_{z}\right)=-\frac{\partial P}{\partial r}+\frac{1}{r} \frac{\partial}{\partial r}\left(2 r \mu \frac{\partial v_{r}}{\partial r}\right) \\
+\frac{\partial}{\partial z}\left[\mu\left(\frac{\partial v_{z}}{\partial r}+\frac{\partial v_{r}}{\partial z}\right)\right]-2 \mu \frac{v_{r}}{r^{2}}-j_{z} B_{\theta} .
\end{gathered}
$$

Axial momentum conservation:

$$
\begin{gathered}
\frac{1}{r} \frac{\partial}{\partial r}\left(r \rho v_{r} v_{z}\right)+\frac{\partial}{\partial z}\left(\rho v_{z}^{2}\right)=-\frac{\partial P}{\partial z}+\frac{\partial}{\partial z}\left(2 \mu \frac{\partial v_{z}}{\partial z}\right) \\
+\frac{1}{r} \frac{\partial}{\partial r}\left[r \mu\left(\frac{\partial v_{z}}{\partial r}+\frac{\partial v_{r}}{\partial z}\right)\right]+j_{i} B_{\theta}
\end{gathered}
$$

Energy conservation:

$$
\begin{gathered}
\frac{1}{r} \frac{\partial}{\partial r}\left(r \rho c_{\mathrm{p}} v_{r} T\right)+\frac{\partial}{\partial z}\left(\rho c_{\mathrm{p}} v_{z} T\right)=\frac{1}{r} \frac{\partial}{\partial r}\left(r k \frac{\partial T}{\partial r}\right)+\frac{\partial}{\partial z}\left(k \frac{\partial T}{\partial z}\right) \\
+\frac{j_{r}^{2}+j_{z}^{2}}{\sigma}+\frac{5 k_{B}}{2 e}\left(j_{r} \frac{\partial T}{\partial r}+j_{z} \frac{\partial T}{\partial z}\right)-U
\end{gathered}
$$

where the subscripts $r$ and $z$ denote radial and axial directions, respectively. The terms in the panes are special momentum and energy source terms concerning with plasma. In Eqs. (1) (4), the basic variables defined are temperature $T$, pressure $P$, radial velocity $v_{r}$ and axial velocity $v_{z}$; the plasma property functions are density $\rho$, viscosity $\mu$, specific heat $c_{\mathrm{p}}$, thermal conductivity $k$ and electrical conductivity $\sigma$, which are all the functions of both temperature and pressure ${ }^{[10]}$. The current density components $j_{r}, j_{z}$ and the magnetic inductive intensity $B_{\theta}$ required in Eqs. (3) and (4) are obtained by Maxwell's equations described in the magnetic vector potential format:

$$
\left\{\begin{array}{l}
\mathbf{E}=-\nabla \varphi-\frac{\partial \mathbf{A}}{\partial t} \\
\mathbf{B}=\nabla \times \mathbf{A}
\end{array}\right.
$$

Plasma Science and Technology, Vol.13, No.2, Apr. 2011

where $\mathbf{E}, \varphi, \mathbf{B}, \mathbf{A}$ are electrical field vector, electrical potential, magnetic inductive intensity vector, magnetic vector potential. Under the steady and axially symmetric conditions, the governing equations can be derived as

Current continuity equation:

$$
\frac{1}{r} \frac{\partial}{\partial r}\left(r \sigma \frac{\partial \varphi}{\partial r}\right)+\frac{\partial}{\partial z}\left(\sigma \frac{\partial \varphi}{\partial z}\right)=0
$$

Ampere's law $\left(\mu_{0}=4 \pi \times 10^{-7} \mathrm{TmA}^{-1}\right)$ :

$$
\left\{\begin{array}{l}
\frac{1}{r} \frac{\partial}{\partial r}\left(r \frac{\partial A_{z}}{\partial r}\right)+\frac{\partial}{\partial z}\left(\frac{\partial A_{z}}{\partial z}\right)=-\mu_{0} j_{z} \\
\frac{1}{r} \frac{\partial}{\partial r}\left(r \frac{\partial A_{r}}{\partial r}\right)+\frac{\partial}{\partial z}\left(\frac{\partial A_{r}}{\partial z}\right)=-\mu_{0} j_{r} .
\end{array}\right.
$$

Ohm's law:

$$
\left\{\begin{array}{l}
j_{z}=-\sigma \frac{\partial \varphi}{\partial z} \\
j_{r}=-\sigma \frac{\partial \varphi}{\partial r} .
\end{array}\right.
$$

For converting A to B, necessary in Eqs. (2) and (3), a component of Eq. (5) is written as

$$
B_{\theta}=\frac{\partial A_{r}}{\partial z}-\frac{\partial A_{z}}{\partial r} .
$$

\subsection{Boundary conditions and numerical method}

The calculation domain of the DC arc plasma torch is shown in Fig. 1, which is a schematic diagram of the experimental torch designed by PAN and the coworkers ${ }^{[8,9]}$. The cathode is made of copper on the base and tungsten alloy on the tip. The anode is made of copper. Argon or gas mixture is chosen as the working gas for plasma. The inter-electrode insert is a part of the torch conventionally designed, which makes the arc column longer and enhances the stability of the arc.

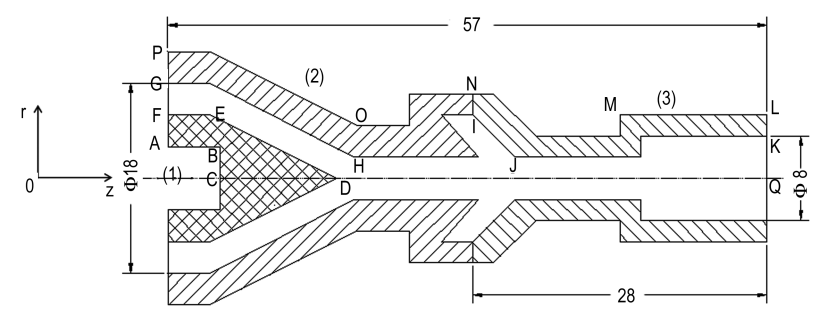

(1) Cathode, (2) Interelectrode insert, (3) Anode

Fig.1 Chematic drawing of the arc plasma torch model (in $\mathrm{mm}$ )

The boundary conditions used in the present modeling are listed in Table 1 with

$$
\gamma \equiv \partial \varphi /(\partial \mathbf{n})=0
$$

and

$$
\Omega \equiv \text { fluid-solid coupled boundary. }
$$

The working gas is axially injected into the torch from the inlet (GF in Fig. 1), then ionized into plasma in the arc zone, which flows out of the outlet (KQ in 
Fig. 1). It is assumed that external surface of the interelectrode insert and the anode (NOP, LMN in Fig. 1) are water-cooled to $500 \mathrm{~K}$, and the root of cathode $(\mathrm{AF}$ in Fig. 1) is assumed to be kept at $1000 \mathrm{~K}$. The boundary condition of Eq. (6) is set by the current density at the root of cathode, while the current density is equal to the value of the total electric current $I$ divided by the area of the cathode root (A_root in Table 1$)$. At the external surface of anode, electrical potential equals to zero. The boundary conditions imposed on the components of the magnetic vector potential are as conventional ${ }^{[5]}$ and, although not being mathematically rigorous, do not violate Ampere's law in Eq. (6). The outflow boundary conditions are used at the outlet of the torch where the flow is regarded as fully-developed. The fluid-solid boundary applied at the interfaces between the electrodes and fluid (FED, IJK in Fig. 1) is the main feature of this modeling study. Through the coupled treatment, both the cathode and anode will be included in the calculation domain, which can avoid to assume boundary conditions at the interface between the electrodes and fluid, with a detailed discussion in section 3.4. In this work, fourteen cases listed in Table 2 are chosen to be simulated, which are with different electric currents, mass flow rates and flow conditions (laminar or turbulent). Under the fixed electric current condition, the flow condition of the torch will transit from laminar to turbulent as the mass flow rate increases ${ }^{[2]}$. The standard $K-\varepsilon$ model is used to simulate the turbulent characteristics of plasma. The con- stants in $K-\varepsilon$ model adopted in this study are taken from Refs. $[3,5,6]$.

All the governing Eqs. (1) (4), (6) and (7) in section 2.2, as typical partial differential equations (PDE), could be solved by using commercial CFD software FLUENT. In addition to the basic fluid-dynamic equations (Eqs. $(1) \sim(4)$ ) contained in FLUENT solver, Eqs. (6) and (7) have to be added into FLUENT solver by using user-defined scalar (UDS) method ${ }^{[11]}$. The special source terms in Eqs. (2) (4) are treated by userdefined function (UDF) method. A structured grid and the semi-implicit method for pressure linked equations (SIMPLE) algorithm are used to solve the governing equations.

\section{Results and discussion}

\subsection{Physics fields inside the plasma torch}

Through the coupled iterative calculation of Eqs. (1) (9), the simulation results can be obtained. Fig. 2 shows the distributions of current density, temperature and velocity inside the plasma torch for arc current $I=170 \mathrm{~A}$ and argon mass flow rate $\dot{m}=$ $1.7 \times 10^{-4} \mathrm{~kg} / \mathrm{s}$. The arc column is clearly shown in Fig. 2(a). The argon gas entering from the inlet is heated and ionized by the Joule heat produced in the arc zone, then driven to accelerate by the electromag-

Table 1. Boundary conditions adopted for the simulation of arc plasma torch

\begin{tabular}{ccccccccc}
\hline & GF & AF & DEF & GHI & NOP & IJK & LMK & KQ \\
\hline$T(\mathrm{~K})$ & 300 & 1000 & $\Omega$ & $\Omega$ & 500 & $\Omega$ & 500 & $\gamma$ \\
$\varphi$ & $\gamma$ & $-\sigma \frac{\partial \varphi}{\partial z}=\frac{I}{A-\text { root }}$ & $\Omega$ & $\gamma$ & $\gamma$ & $\Omega$ & 0 & $\gamma$ \\
$A_{i}$ & $\gamma$ & $\gamma$ & $\Omega$ & $\gamma$ & $\gamma$ & $\Omega$ & $\gamma$ & 0 \\
$v_{i}$ & $\rho v_{z}=\frac{\dot{m}}{A_{-} \text {inlet }}$ & - & 0 & 0 & - & 0 & - & $\gamma$ \\
\hline
\end{tabular}

Table 2. Operational conditions for the simulated cases

\begin{tabular}{cccc}
\hline Case & Flow condition & Electric current $(\mathrm{A})$ & Mass flow rate $\left(10^{-4} \mathrm{~kg} / \mathrm{s}\right)$ \\
\hline 1 & Laminar & 170 & 1.7 \\
2 & Laminar & 170 & 1.9 \\
3 & Laminar & 170 & 2.1 \\
4 & Laminar & 170 & 2.3 \\
5 & Turbulent & 170 & 3.6 \\
6 & Turbulent & 170 & 3.8 \\
7 & Turbulent & 170 & 4.0 \\
8 & Turbulent & 170 & 4.2 \\
9 & Laminar & 170 & 1.7 \\
10 & Laminar & 190 & 1.7 \\
11 & Laminar & 210 & 1.7 \\
12 & Turbulent & 170 & 3.8 \\
13 & Turbulent & 190 & 3.8 \\
14 & Turbulent & 210 & 3.8 \\
\hline
\end{tabular}

Cases $1 \sim 8$ with the same total current and different mass flow rates, Cases $9 \sim 14$ with different total current and mass flow rates 
netic field. The arc voltage is important but hard to be predicted in the simulation. The calculated arc voltage is often far from the experimental value, especially in the 2D simulation ${ }^{[3]}$. However, as shown in Fig. 3, the calculated arc voltage in this work is very close to the experimental value ${ }^{[12]}$. This will be discussed in section 3.4 .
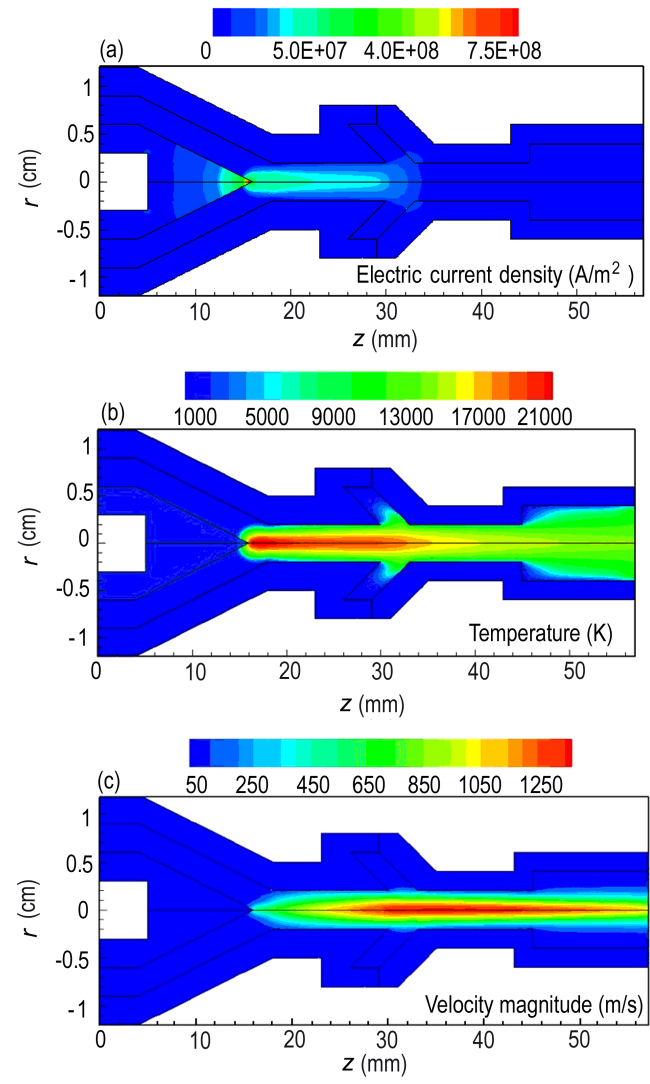

(a) Current density, (b) Temperature, (c) Velocity magnitude

Fig.2 Calculated results of physical fields for argon plasma torch $\left(170 \mathrm{~A}, 1.7 \times 10^{-4} \mathrm{~kg} / \mathrm{s}\right)$

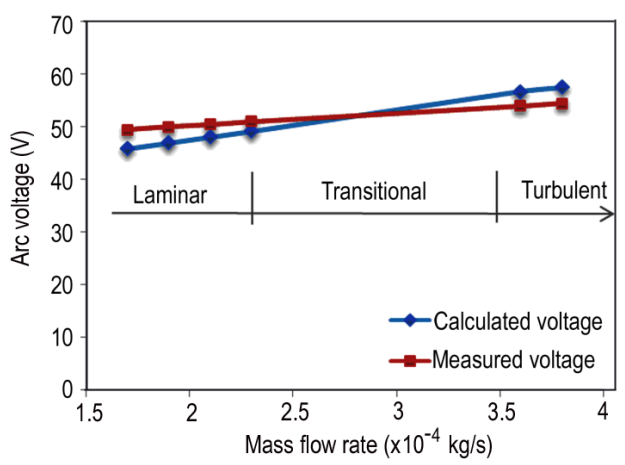

Fig.3 Calculated and measured arc voltage for the torch (170 A) as functions of mass flow rate
Plasma Science and Technology, Vol.13, No.2, Apr. 2011

The result shown in Fig. 3 indicated that the arc voltage increases linearly as the mass flow rate increases while the arc current is unchanged, no matter the flow condition is laminar or turbulent. Because the $K-\varepsilon$ model is just suitable for modeling the turbulence with a high Reynolds number rather than the transitional process, the laminar and turbulent conditions are simulated by the laminar model and the $K-\varepsilon$ model, respectively. The arc voltages calculated by using laminar and turbulent model are consistent with the experiment results shown in Fig. 3, respectively. By drawing a line through the points of calculated results, the situation of the transitional process can be roughly predicted, which is also consistent in trend with the experiment results.

\subsection{Outlet parameters of the torch and the influence of mass flow rate and electric current}

As listed in Table 3, the calculated results on temperature and velocity at the outlet are consistent with the measured results ${ }^{[12]}$, in which $U_{\max }, \bar{U}, T_{\max }$ and $\bar{T}$ represent the maximum velocity, mass averaged velocity, maximum temperature, mass averaged temperature, respectively.

The calculated radial distributions of temperature and velocity at the outlet of the plasma torch are shown in Fig. 4 for Case $1 \sim 6$, and Fig. 5 for Case $9 \sim 14$. It is
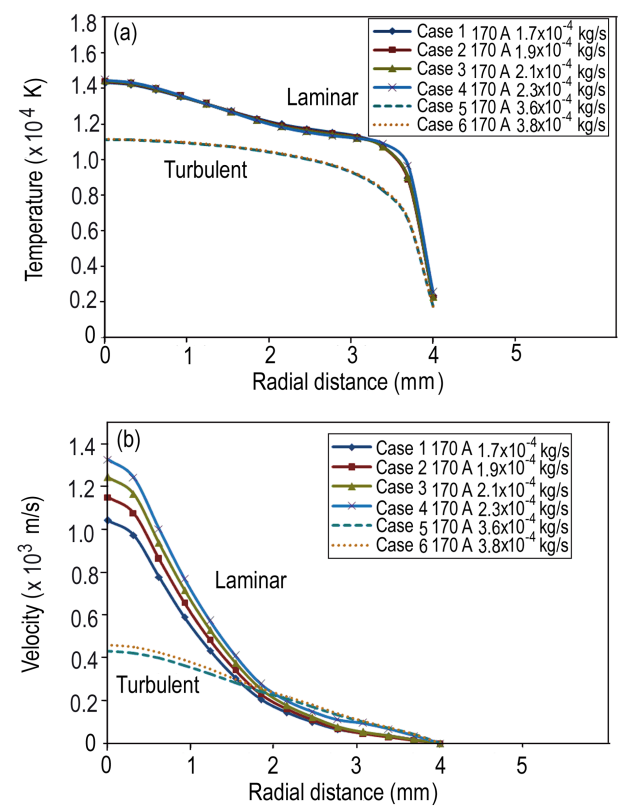

(a) Temperature, (b) Velocity

Fig.4 Radial distributions of temperature and velocity at the outlet of the plasma torch for the same total current and different mass flow rates (case $1 \sim 6$ )

Table 3. Comparison of calculated results and experimental ones of the torch $\left(170 \mathrm{~A}, 1.7 \times 10^{-4} \mathrm{~kg} / \mathrm{s}\right)$

\begin{tabular}{lcccc}
\hline & $U_{\max }(\mathrm{m} / \mathrm{s})$ & $\bar{U}(\mathrm{~m} / \mathrm{s})$ & $T_{\max }(\mathrm{K})$ & $\bar{T}(\mathrm{~K})$ \\
\hline Calculated results Experimental results $^{[10]}$ & 1042 & 432 & 14338 & 12834 \\
& 900 & 450 & 16000 & 14000 \\
\hline
\end{tabular}


DENG Jing et al.: Numerical Simulation of Fluid Flow and Heat Transfer in a DC Non-Transferred Arc
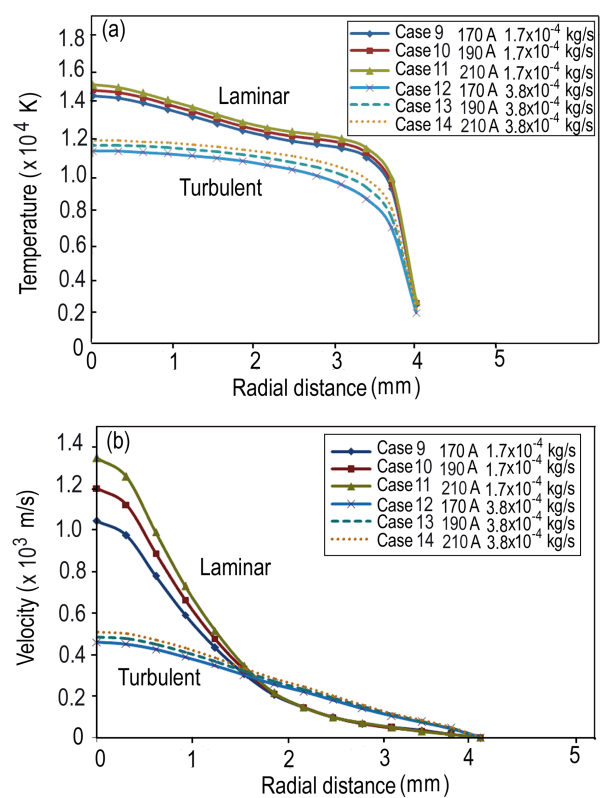

(a) Temperature, (b) Velocity

Fig.5 Radial distributions of temperature and velocity at the outlet of the plasma torch for the same mass flow rates and different total current (case 9 14)

revealed that the maximum temperature and velocity always appear at the axis of the torch outlet, and the distributions of temperature and velocity are flatter in the turbulent flow condition than that in laminar one. In Fig. 4(a) it is indicated that the temperature distributions at the outlet are unchanged under the same current condition even if the mass flow rates change, but they stay at two different levels under the laminar and turbulent flow condition, respectively. This interesting phenomenon has also been observed in a related experiment ${ }^{[12]}$. That's because the arc voltage linearly increases with the increase in mass flow rate with a fixed arc current keeps, as shown in Fig. 3, thereby the input electric power also linearly increases with the increase in mass flow rate. Then the input power will be transferred into the Joule heat which uniformly heats the gas when the anodic arc root attachment circumferentially and uniformly distributes inside the torch. Fig. 4(b) shows that the velocity increases as the mass flow rate increases, which satisfies the requirement of mass conservation. Fig. 5 shows that the temperature and velocity increase with the increase in total current, because the Joule heat will be enhanced as the total current increases, then the working gas will be heated more strongly.

\subsection{Thermal efficiency of the torch and the influence of mass flow rate and electric current}

The thermal efficiency of the torch $\eta$ is defined as the ratio of the total enthalpy of the high temperature gas at the outlet of the torch to the total electric power input, i.e.

$$
\eta=\frac{H_{\mathrm{outlet}}}{V_{\mathrm{arc}} \cdot I}
$$

where $H_{\text {outlet }}, V_{\text {arc }}$ and $I$ represent total enthalpy of the high temperature gas at the outlet, arc voltage and total current, respectively. Similarly, define $\xi_{\text {Cathode }}, \xi_{\text {Anode }}$ and $\xi_{\text {Interelectride }}$ as

$$
\begin{aligned}
\xi_{\text {Cathode }} & =\frac{Q_{\text {Cathode }}}{V_{\text {arc }} \cdot I}, \\
\xi_{\text {Anode }} & =\frac{Q_{\text {Anode }}}{V_{\text {arc }} \cdot I}, \\
\xi_{\text {Interelectrode }} & =\frac{Q_{\text {Interelectrode }}}{V_{\text {arc }} \cdot I},
\end{aligned}
$$

to represent the thermal loss coefficients of the cathode, anode and inter-electrode insert, which mean the fractions of the heat taken from the cathode, anode and interelectrode insert by cooling water, respectively. $Q_{\text {Cathode }}, Q_{\text {Anode }}$, and $Q_{\text {Interelectrode }}$ can be calculated by the integration of the heat flux at the water-cooled surfaces of the three parts. With the previous calculated $V_{\text {arc }}$ and $I$, the solutions of Eqs. (10) and (11) are obtained and shown in Fig. 6. The mass flow rate and current are thought as the main parameters to affect the thermal efficiency of the torch in this study. In the thermal efficiency analysis of the arc plasma torches, the arc length is usually regarded as one of the main parameters ${ }^{[5,13]}$. However, the interelectrode insert of the simulated torch is a specially-designed part that can make the arc length of the torch much longer than that of torches without an interelectrode insert. Hence, the influence of the variation of arc length can be neglected in this case.

Fig. 6(a) and (b) show the influence of mass flow rate on the thermal efficiency of the torch. It can be found that the thermal efficiency is much higher in a laminar torch than in a turbulent one. Under both the laminar and turbulent conditions, the thermal efficiency will increase with the increase in mass flow rates. The maximum thermal efficiency will occur when the flow condition is about to transit. According to the difference between the laminar and turbulent results, it could be assumed that the thermal efficiency will dramatically decrease in the transitional process. Fig. 6(c) and (d) show the influence of the total current on the thermal efficiency of the torch. It can be found that the thermal efficiency will decrease with the increase in total current. Fig. 6 also shows that most of the thermal loss comes from the water-cooled surface of the anode, because the arc root is attached at the inner surface of the anode, the current will cause a lot of Joule heat near the inner surface of the anode. Moreover, the area of the water-cooled surface of the anode is much larger than the cathode's, so most of the heat will be taken from the anode by cooling water.

Based on the above discussion, it can be concluded that a torch operated in the laminar condition with low current and high mass flow rate could be with better thermal efficiency. Moreover, the surface area of the anode should be strictly limited in the design of the torch. 

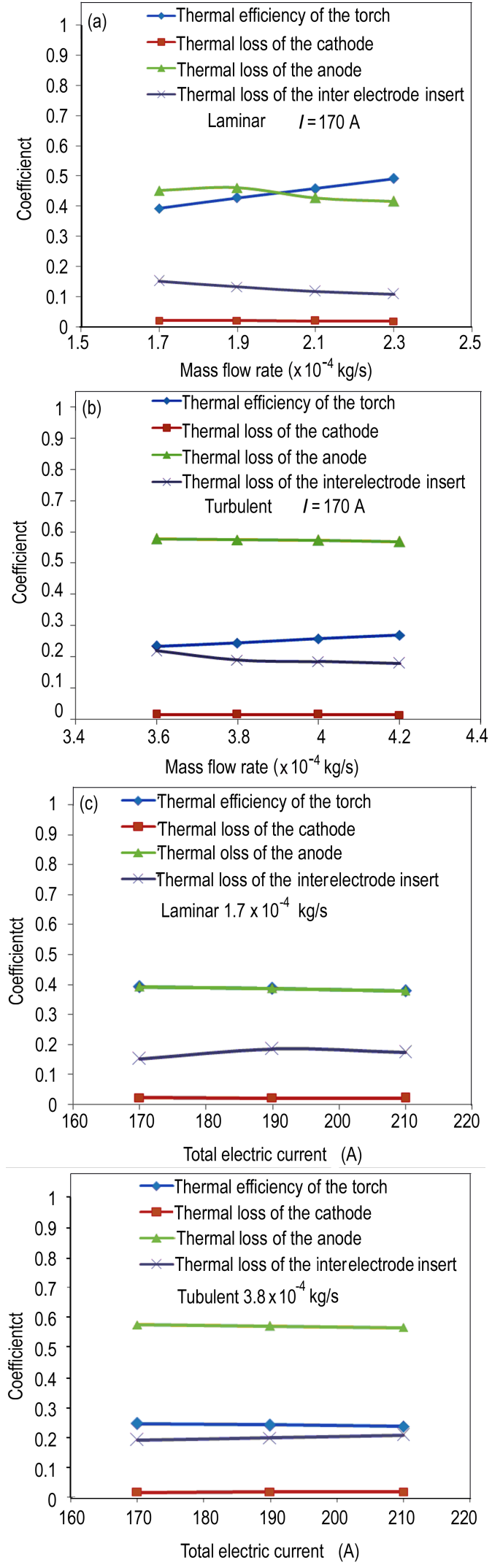

(a) Influence of the mass flow rate (laminar, $170 \mathrm{~A}$ ), (b) Influence of the mass flow rate (turbulent, $170 \mathrm{~A}$ ), (c) Influence of the total current (laminar, $1.7 \times 10^{-4} \mathrm{~kg} / \mathrm{s}$ ), (d) Influence of the total current (turbulent, $3.8 \times 10^{-4} \mathrm{~kg} / \mathrm{s}$ )

Fig.6 Influence of mass flow rate and total current on both the thermal efficiency of the torch and the thermal loss coefficients of each part of the torch
Plasma Science and Technology, Vol.13, No.2, Apr. 2011

\subsection{Fluid-solid coupled method and the prediction of anodic arc root loca- tion}

Thermal deformation and erosion at the arc root attachment of the electrodes extremely reduce the service life of plasma torches. So the prediction of the location of arc root attachment is particularly important. Some boundary conditions are usually assumed to limit the location of arc root attachment at the internal surface of anode. In this work, the arc root attachment is automatically calculated without any limiting treatment for the boundary condition at the electrodes. The axial distribution of the current density at the internal surface of the inter-electrode insert and anode is shown in Fig. 7 where the color lines correspond to different parts of the internal surface of the torch marked in Fig. 1. The maximum current density in Fig. 7 corresponds to arc root attachment at the internal surface of anode. The predicted location is exactly consistent with the location observed in the experiment ${ }^{[2]}$.

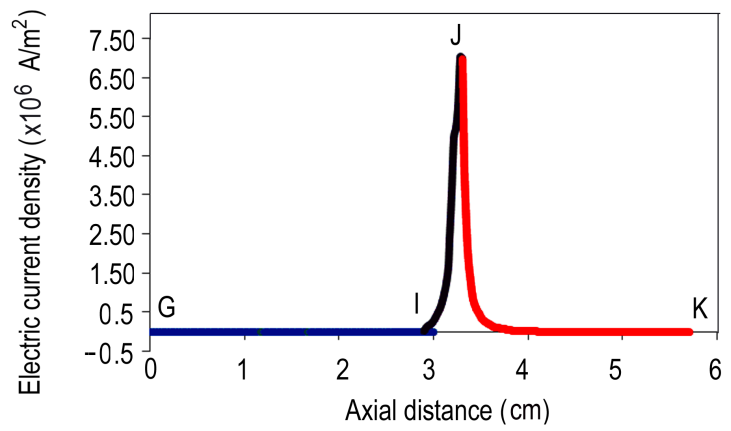

Fig.7 Axial distribution of current density in the internal surface of the plasma torch $\left(170 \mathrm{~A}, 1.7 \times 10^{-4} \mathrm{~kg} / \mathrm{s}\right)$

Because a two-dimension (2D) numerical model is used, the calculated result shown in Fig. 2(a) indicates the formation of a circumferentially uniform arc root attachment. Known from the experimental experience, the circumferentially uniform arc root attachment actually exists under the operational condition of low current and single species of working gas. However, for high current or multi-species for working gas, the arc root attachment will be circumferentially non-uniform, which means that the arc root will be concentrated at a spot on the internal surface of the anode. In the future study, a 3D numerical model will be applied to simulate the phenomenon of the concentrated arc root.

There are two reasons which lead to the accurately calculated results in this study. Firstly, the 2D model is used in the right situation when the anodic arc root attachment in the experiment is circumferentially uniform, which also means that it is a $2 \mathrm{D}$ distribution. Secondly, the fluid-solid coupled boundary conditions are applied to the interfaces between the electrodes and fluid. The use of coupled boundary condition is of great advantages. It can guarantee the continuity of both current and heat flux between the electrodes and fluid, avoiding the assumed boundary condition imposed on the internal surface of anode ${ }^{[6,7]}$. It can 
also avoid to impose the boundary condition for heat flux and current on the tip of cathode, which used to be a usual way to treat the boundary condition of the cathode ${ }^{[3,5,6]}$. In this work, the average current density is given at the root of the cathode (AF in Fig. 1), which could avoid giving the assumed current density at the cathode tip ${ }^{[3,5,6]}$. Moreover, the assumed maximum current density adopted in Refs. [3] and [6] are $4.0 \mathrm{E}+8 \mathrm{~A} / \mathrm{m}^{2}$ for $I=400 \mathrm{~A}$ and $7.0 \mathrm{E}+7 \mathrm{~A} / \mathrm{m}^{2}$ for $I=500$ A respectively, which is even smaller than the calculated one under a much lower total current condition in this study, such as $8.0 \mathrm{E}+8 \mathrm{~A} / \mathrm{m}^{2}$ for $I=170 \mathrm{~A}$ shown in Fig. 2(a). The assumed temperature distributions at the cathode tip adopted in Refs. [3] and [6] are reasonable and close to the calculated one in this study, as shown in Fig. 2(b). By using fluid-solid coupled method in the calculation of electromagnetic field, the location of anodic arc root attachment is automatically predicted rather than being limited in the treatment of electromagnetic boundary conditions ${ }^{[6]}$.

\section{Conclusions}

In this work, a magnetic fluid dynamics (MHD) model with vector potential description and fluid-solid coupled method is used to simulate the electromagnetic field, heat transfer and fluid flow in a DC nontransferred arc plasma torch. The main conclusions drawn from the present study are as follows.

a. The 2D MHD model is suitable for simulation when the arc root attachment is circumferentially uniform as in the experiment, But the concentrated arc root phenomenon must be simulated by using a $3 \mathrm{D}$ MHD model.

b. The fluid-solid coupled boundary conditions for the equations of electromagnetic field and energy conservation are crucial and necessary to guarantee the continuity of both current and heat flux between the electrodes and fluid. Through the fluid-solid coupled method one can also avoid to impose any assumed electric and thermal conditions on the cathode tip, and obtain more accurate arc voltage and location of the anodic arc root attachment.

c. The arc voltage increases linearly with the increase in mass flow rate while the total arc current keeps unchanged, which cause the temperature distributions at the torch outlet unchanged under the same current while the mass flow rates change, but the temperature distributions would keep at two different levels under the laminar and turbulent flow condition, respectively.

d. A torch operated under the laminar condition with low current and high mass flow rate could be with better thermal efficiency, and, it could be speculated that the thermal efficiency will dramatically decrease in the transitional process. Most of the thermal loss is from the water-cooled surface of the anode. Hence, the surface area of the anode should be strictly limited in the design of plasma torches.

\section{Acknowledgements}

Grateful acknowledgement is to Professor PAN Wenxia from the Institute of Mechanics, Chinese Academy of Sciences, for her suggestions about this study.

\section{References}

1 Li Yaojian, Huang Zhiqin, Xu Yongxiang, et al. 2009, Environ. Eng. Sci., 26: 731

2 Pan Wenxia, Meng Xian, Chen Xi, et al. 2006, Plasma Chem. Plasma Process., 26: 335

3 Li Heping, Chen Xi. 2001, J. Phys. D: Appl. Phys., 34: 99

4 Jing Deng, Li Yaojian, Sheng Hongzhi, et al. 2010, Journal of Engineering Thermophysics, 31: 879 (in Chinese)

5 Trelles Juan Pablo, Pfender Emil, Heberlein Joachim. 2006, Plasma Chem. Plasma Process., 26: 557

6 Selvan B, Ramachandran K, Sreekumar K P, et al. 2010, Vacuum, 84: 444

7 Hsu K C, Etemadi K, Pfender E. 1983, J. Appl. Phys. 54: 1293

8 Pan W X, Zhang W H, Zhang W H, et al. 2001, Plasma Chem. Plasma Process., 21: 23

9 Pan W X, Zhang W H, Ma W, et al. 2002, Plasma Chem. Plasma Process., 22: 271

10 Murphy A B, Arundell C J. 1994, Plasma Chemistry and Plasma Processing, 14: 451

11 Fluent Inc. 2005, Fluent 6.2 User-Defined Functions Manual, Lebanon, NH

12 Meng Xian, Pan Wenxia, et al. 2004, Journal of Engineering Thermophysics, 25: 490 (in Chinese)

13 Li Heping, Pfender E, Chen Xi. 2003, Journal of Physics D: Applied Physics, 36: 1084

(Manuscript received 31 May 2010)

(Manuscript accepted 12 October 2010)

E-mail address of DENG Jing: dengjing@imech.ac.cn 